\title{
Experimental Verification of Formulae for Natural Seiche Frequencies in a Two-layer Fluid with the Free Surface
}

\author{
Victor Ivanovich Bukreev (Corresponding author) \\ Lavrentyev’ Institute of Hydrodynamics, Siberian Branch of Russian Academy of Sciences \\ 15 Lavrentyev’ av., Novosibirsk, 630090, Russia \\ E-mail: bukreev@hydro.nsc.ru
}

\begin{abstract}
Victor Vasilievich Zykov
Lavrentyev’ Institute of Hydrodynamics, Siberian Branch of Russian Academy of Sciences

15 Lavrentyev’ av., Novosibirsk, 630090, Russia

E-mail: bukreev@hydro.nsc.ru
\end{abstract}

\begin{abstract}
Alexey Vladimirovich Chebotnikov
Lavrentyev' Institute of Hydrodynamics, Siberian Branch of Russian Academy of Sciences

15 Lavrentyev’ av., Novosibirsk, 630090, Russia

E-mail: bukreev@hydro.nsc.ru
\end{abstract}

Received: January 5, 2013 Accepted: February 3, 2013 Published: July 1, 2013

doi:10.5296/ast.v1i2.3258ＵRL: http://dx.doi.org/10.5296/ast.v1i2.3258

\begin{abstract}
Theoretical formulae for the natural frequencies of oscillations of a two-layer fluid due to surface and interface seiches are experimentally tested. It is shown that the formulae based on the strict dispersion relation of the linear theory of the potential motion are applicable in a wide range of the natural frequencies. The approximate formulae based on the long-wave approximation are also applicable but in a narrower frequency range, which is particularly typical for the natural seiches.
\end{abstract}


Keywords: Two-layer fluid, Surface and internal seiches, Natural modes, Frequency spectrum, Attenuation coefficient 


\section{Introduction}

A seiche is a standing wave in an enclosed or partially enclosed body of water (http://en.wikipedia.org/wiki/Seiche). Seiches generate significant horizontal and vertical velocities of water movements. It is unpleasant for ships and is essential for ecology. Both surface and internal seiches exist in water with stable density stratification. There are the explicit theoretical formulae for the wavelengths and frequencies of the different seiche modes in the case of homogeneous initially quiescent fluid filling a rectangular basin with the flat horizontal bottom (Kochin et al., 1963). The linear theory of the potential motion of fluid is used in the theory so the fluid viscosity is not taken into account. The results of an experimental verification of these formulae are contained in Bukreev (2011). The empirical formulae for surface seiches in basins of more complex forms may be found in Rabinovich, (2009).

The explicit formulae are available in Phillips (1967) and Dotsenko and Miklashevskaya (2010) or can also be derived (see below) in the case of a two-layer fluid. Only a two-layer fluid under a horizontal cover was considered in Phillips (1967). The so-cold long wave approximation in the framework of the cited theory was used in Dotsenko \& Miklashevskaya, (2010). The theories give relative amplitudes of seiches only. Particular values of the amplitudes depend on the type of disturbance and its intensity. Verification of the theoretical formulae for the frequency oscillations due to surface and internal seiches in a two-layer fluid in controlled laboratory conditions was the main aim of the present work.

Natural seiches are generated by wind and atmospheric pressure variations, seismic activity, tsunamis, landslides, etc. The total property of these disturbances is their relative brevity. As the results, progressive waves are generated at first (Boegman et al., 2005). Seiches are born after the reflection of the progressive waves and are superimposed on the last. As a rule, the same properties of perturbations and waves are typical for laboratory experiments. This is also true for the present work, in which the waves were generated just as it has been done in the experiments of Thorpe (1971); Horn et al. (2001); Boegman (2004); Boegman et al. (2005). Only internal waves were studied in these experiments. Thorpe (1971) received the classical patterns of the shear instability in a two-layer fluid. Progressive internal surges in the form of the moving hydraulic jump were the main object of attenuation in the experiment of Horn et al. (2001); Boegman (2004).

Both surface and internal seiches are specially extracted in the present experiments and theoretical formulae for the wavelengths and frequencies of seiche oscillations are revised on this refined base. That is the main novelty of the present paper.

\section{Theoretical Formulae}

The wave numbers $k_{p}$ of the set of the longitudinal seiches in a basin with horizontal bottom are determined by the following formula:

$$
k_{p}=\pi p / L
$$


where $p=1,2,3, \ldots$ is the number of the normal mode, $L$ is the basin length. The corresponding oscillation frequencies $\omega_{p}$ can be obtained from the dispersion relation $\omega(k)$ of the linear theory, assuming $k=k_{p}$ in the relation ( $k$ is an arbitrary wave number, $\omega$ is the corresponding circular frequency). The strict equation for $\omega(k)$ in the problem considered has the form (Ovsyannikov et al., 1985):

$$
(k g)^{2}\left(1+\gamma \Theta_{1} \Theta_{2}\right) \omega^{4}-k g\left(\Theta_{1}+\Theta_{2}\right) \omega^{2}+\Theta_{1} \Theta_{2}(1-\gamma)=0
$$

where $g$ is the acceleration due to gravity,

$$
\Theta_{1}=\tanh \left(k h_{1}\right), \Theta_{2}=\tanh \left(k h_{2}\right), \gamma=\rho_{1} / \rho_{2}
$$

$h_{1}$ and $h_{2}$ are the depths of the upper and lower layers, $\rho_{1}, \rho_{2}$ are the densities of those layers. Equation (1) at each $k=k_{p}$ has four roots. The sign of the root determines the direction of wave propagation. Only two positive roots have a physical meaning for standing waves, namely:

$$
\begin{gathered}
\omega 1_{p}=\sqrt{\left[\frac{1}{2} b_{p}+\left(\frac{1}{4} b_{p}^{2}-c_{p}\right)^{0.5}\right] k_{p} g} \\
\omega 2_{p}=\sqrt{\left.\frac{1}{2} b_{p}-\left(\frac{1}{4} b_{p}^{2}-c_{p}\right)^{0.5}\right] k_{p} g}
\end{gathered}
$$

where

$b_{p}=\frac{\Theta 1_{p}+\Theta 2_{p}}{1+\gamma \Theta 1_{p} \Theta 2_{p}}, \quad c_{p}=\frac{(1+\gamma) \Theta 1_{p} \Theta 2_{p}}{1+\gamma \Theta 1_{p} \Theta 2_{p}}$,

Formulae (3) and (4) determine the natural frequencies of the surface and internal oscillations, accordingly.

When hyperbolic tangent in equation (3) can be replaced by its argument (that is the long wave approximation), the following approximate formulae are obtained in Dotsenko and Miklashevskaya, (2010):

$$
\begin{aligned}
& \omega 1_{p} \approx \sqrt{\frac{2 \varepsilon g h_{1} h_{2} p^{2} \pi^{2}}{L^{2}\left(H-\sqrt{H^{2}-4 \varepsilon h_{1} h_{2}}\right)}} \\
& \omega 2_{p} \approx \sqrt{\frac{2 \varepsilon g h_{1} h_{2} p^{2} \pi^{2}}{L^{2}\left(H+\sqrt{H^{2}-4 \varepsilon h_{1} h_{2}}\right)}}
\end{aligned}
$$

where $H=h_{1}+h_{2}, \quad \varepsilon=1-\rho_{1} / \rho_{2}=1-\gamma$.

If the density stratification is weak ( $\varepsilon<<1$ ), formulae (6) and (7) take the next form (Dotsenko \& Miklashevskaya, 2010): 


$$
\begin{gathered}
\omega 1_{p} \approx \sqrt{\frac{p^{2} \pi^{2} g H}{L^{2}}} \\
\omega 2_{p} \approx \sqrt{\frac{p^{2} \pi^{2} \varepsilon g h_{1} h_{2}}{L^{2} H}}
\end{gathered}
$$

The mutual influence of the surface and internal seiches is not taken into account in formulae (8) and (9). It should be noted that Coriolis acceleration is taken into consideration in Dotsenko and Miklashevskaya (2010). The corresponding term in formulae (6)-(9) is omitted.

When a transition layer with thickness $\delta<<H$ exists between the upper and lower layers, the values $\omega 2_{p}$ can be estimated by the next approximate formula by Phillips (1967):

$$
\omega 2_{p} \approx \sqrt{\frac{\varepsilon g k_{p}}{k_{p} \delta+\operatorname{ctan}\left(k_{p} h_{1}\right)+\operatorname{ctan}\left(k_{p} h_{2}\right)}}
$$

\section{Experiment and Comparison with the Theory}

The diagram of experimental setup and fixed rectangular coordinate system are presented in Figure 1. The rectangular basin made of perspex had the length $L=1.98 \mathrm{~m}$, the width $B=0.06 \mathrm{~m}$ and its bottom was flat. The basin could rotate in vertical direction around the support 5 which was located at the distance $l=1.55 \mathrm{~m}$ from the coordinate system origin (see Figure 1). An analogous setup was used in the experiments of Horn et al. (2001) and Boegman (2004). The basin dimensions were large enough for the similarity of wave processes on the Froude criteria and the self-preservation on the Reynolds criteria to be true. Particularly, this was confirmed by the results of comparison of the received experimental data with the theory in which the Froude criteria is the only similarity criterion (see below).

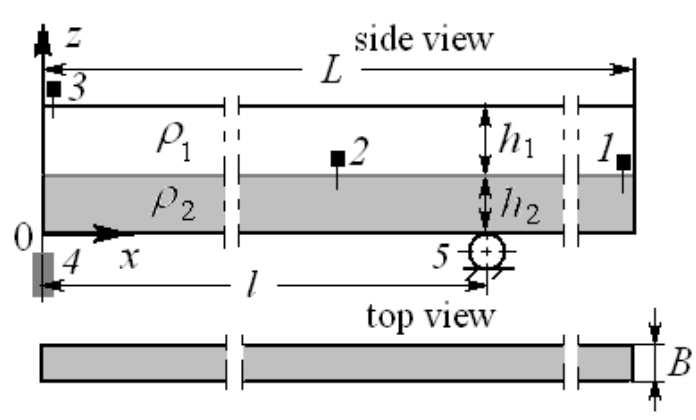

Figure 1. Diagram of the experimental setup

Notes: 1, 2, 3, Wavegauges; 4, Device for rotate; 5, Support.

The basin was firstly filled by a layer of tap water of density $\rho_{1}$ up to $h_{1}$ in depth. After that, a weak glycerin solution with the density $\rho_{2}>\rho_{1}$ was slowly supplied into the basin bottom thus displacing the buoyant fresh water layer. The glycerin solution viscosity exceeded the tap water viscosity no more than $2.5 \%$. The depth of the transition layer $\delta$ 
after the filling of two layers was about $1 \mathrm{~mm}$. Then the basin was rotated upwards by the angle $\beta$ with the help of device 4 (see Figure 1). When any movement of water was stopped, the basin was rotated to the horizontal position to generate surface and internal waves. The angle $\beta$ and the time $\tau$ of the basin displacement to the horizontal position are substantial parameters of the problem. Variation of these parameters allows one to obtain different waves (Boegman, 2004). Such combination of the parameter was most suitable for the aim of the present work when waves are smooth and the relative contribution of progressive waves is small. Below, there are two relevant examples. They will be named as Experiment 1 and Experiment 2.

Vertical oscillations $\zeta$ of the free surface and interface were recorded by the resistive wavegauges 1, 2, and 3 (see Figure 1). The wavegauges could register the oscillations, which exceed $0.2 \mathrm{~mm}$, without substantial errors. Their natural frequency was about $4 \mathrm{~Hz}$. It is shown below that the highest frequencies in the oscillation spectra were less than $1 \mathrm{~Hz}$. The wavegauge 1 was located at the distance of $3 \mathrm{~cm}$ from the right butt end of the basin. It registered internal waves. The wavegauge 2 was located in the basin midst and also registered internal waves. The wavegauge 3 was located at the distance of $3 \mathrm{~cm}$ from the basin left butt end and registered surface waves.

The wavegauges registered the summary oscillations due to all normal modes. The spectral analysis was performed to determine frequencies of individual modes. The algorithm of Fast Fourier Transformation (FFT) of $\zeta$-oscillations was used for this aim. Analysis was performed with the help of the software application Origin computer product. There are two versions of FFT in Origin named as FFT-amplitude and. FFT-energy. The FFT-amplitude version was more appropriate for unsteady deterministic processes, which are considered in the present paper. The FFT-energy version is used in an analysis of steady random processes, particularly in turbulent fluctuations. The energy of internal waves has been considered by Boegman (2004) and Boegman et al. (2005).

Two problems should be solved at a spectral analysis. One problem is a choice of a time interval for the analysis. Fading $\zeta$-oscillations take place in the experiments and a value of time, above which all $\zeta<0.2 \mathrm{~mm}$, was used as the upper boundary of the interval. Second problem is the next. Finiteness of the time interval leads to the fact that the product of $\zeta$ function and the Heaviside step function (but not the function on demand) is analyzed. So-cold smoothing algorithm is used in order to eliminate the corresponding error. The software application Origin offers a choice of five smoothing algorithms. In the case of $\zeta$-oscillations considered, discrete frequencies in spectra was segregated most clearly by using the "Blackman" algorithm,

Synchronous records of the free surface and interface oscillations in Experiment 1 and Experiment 2 are given in Figures 2(a) and 2(b), respectively. Only given parameters $\beta$ and $\tau$ were different in these experiments. Their values are given in the legend to Figure 2. Other parameters, in particular $h_{1}=0.06 \mathrm{~m}, h_{2}=0.04 \mathrm{~m}, \rho_{1}=1000 \mathrm{~kg} / \mathrm{m}^{3}, \quad \rho_{2}=1009.2$ $\mathrm{kg} / \mathrm{m}^{3}$, were unchanged. In Figure 2, time $t$ is referred to the start of the basin movement to the horizontal position. The functions $\zeta_{i}(t)(i=1,2,3)$ are the oscillations which were registered by the wavegauges 1,2 , and 3 , respectively. Over the time, these functions attain constant asymptotic values corresponding to the state of rest. These asymptotic values are equal to zeros in Figure 2. 

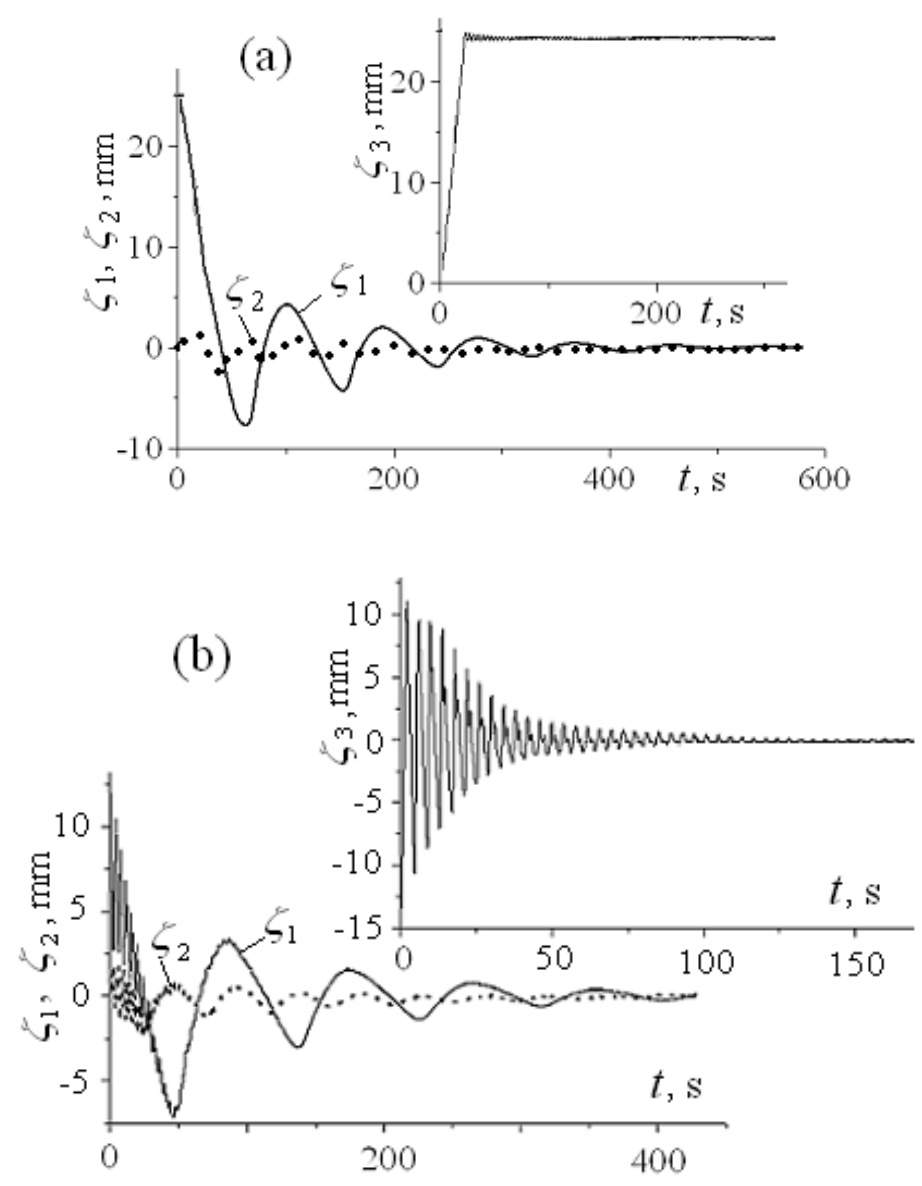

Figure 2. Oscillations of the interface $\left(\zeta_{1}, \zeta_{2}\right)$ and surface $\left(\zeta_{3}\right)$

Notes: (a) Experiment 1: $\beta=1.22^{\circ}, \tau=25 \mathrm{~s}$; (b) Experiment 2: $\beta=0.42^{\circ}, \tau=2 \mathrm{~s}$.

In Experiment 1, the free surface attained monotonically its asymptotical level almost just after the rotation of the basin to the horizontal position and the surface waves were very small. In Experiment 2, the surface waves were significant and affected the internal waves. All seiche modes have the antinodes at the basin ends. As the result, the summary oscillations are maximal here. In the basin middle, all odd seiche modes have the nodes so the oscillations are minimal here. The viscosity of water is not considered in the theory. Data given in Figure 2 showed that the waves were degenerated and the surface waves were degenerated faster than the internal ones.

The influence of the surface waves on the internal ones is illustrated by two images shown in Figure 3. The images are received in Experiment 1 with the values of $\tau=0.3 \mathrm{~s}$ at two $t$ values. The bottom layer is colored with aquatic solution of commercial ink (3 milliliters of commercial ink in 1 liter of water). Solid particles are visible in the images. That is wax (paraffin) weighted by aluminum powder. Some particles are lighter then fresh water and track the movement of the free surface. Other particles have the density near the density of salt water in the lower layer. They tracked the movement in the neighborhood of the 


\section{Macrothink}

interface. It should be noted that the pointed way of preparation of relatively large marked particles was the most successful among a number of others ways. In the case of stable stratified salt water, such particles tracked the corresponding isopycnic surface for a few days. The distance between the vertical solid lines in Figure 3 is equal to $0.2 \mathrm{~m}$. The dash vertical line in Figure 3 makes the position of the node of second normal mode seiche.
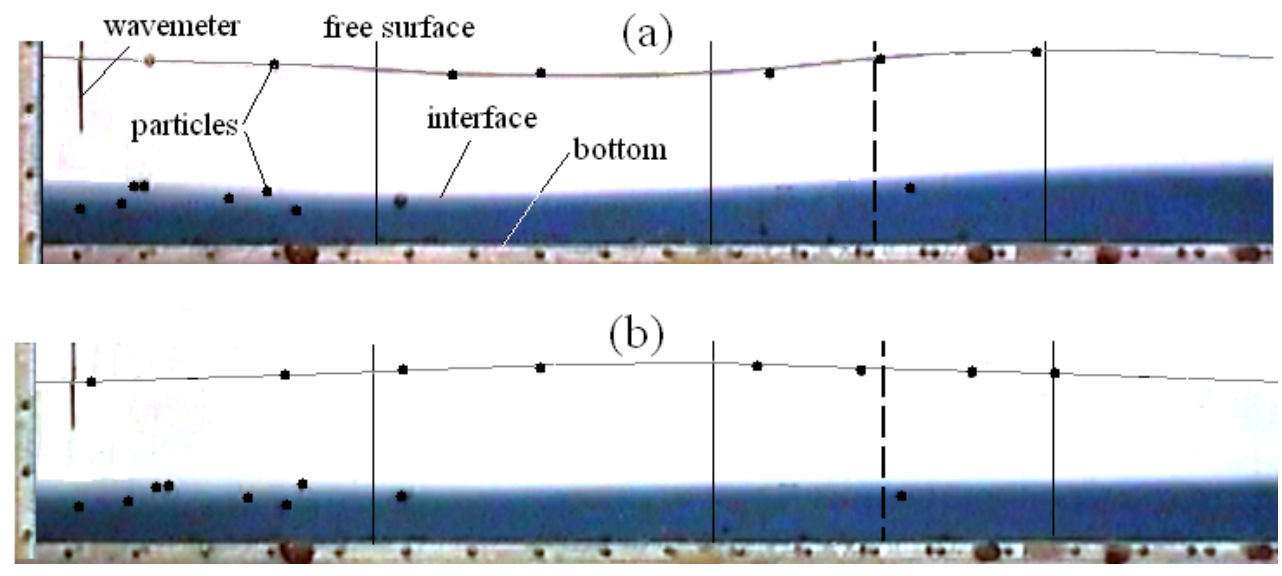

Figure 3. Example of influence of surface wave on internal wave at $\beta=1.22^{\circ}$ and $\tau=0.3 \mathrm{~s}$

Notes: (a) $t=10.3 \mathrm{~s}$; (b) $t=12.3 \mathrm{~s}$.

The dimensionless modules $\left|S_{i}^{0}(f)\right|$ of the spectra $S_{i}(f)$ of the functions $\zeta_{i}(t)$ are presented in Figures 4(a) and 4(b) ( $f$ is a frequency). The maximum of the dimension experimental spectrum at $i=1$ and $j=1$ is used as the scale for all dimensionless spectra. Numerical values of these scales are different in Figure 4(a) and Figure 4(b). The modulus $\left|S_{3}^{0}(f)\right|$ is not shown in Figure 4(a) whereas the surface waves in Experiment 1 were too weak (see $\zeta_{3}$ in Figure 2(a)). The spectra have a number of the maxima at the frequencies $f_{j}^{e}(j=1,2,3, \ldots)$. These frequencies are compared below with the theoretical frequencies of the seiche oscillations. There is a specific maximum $A$ in Figure 4 . It is due to the unsteady nature of the waves and it is not described by the theory. The functions $\left|S_{2}^{0}(f)\right|$ have notable maxima only at the even values of $j$ since the corresponding wavegauge was located in the theoretical nodal point for all odd seiche modes. 

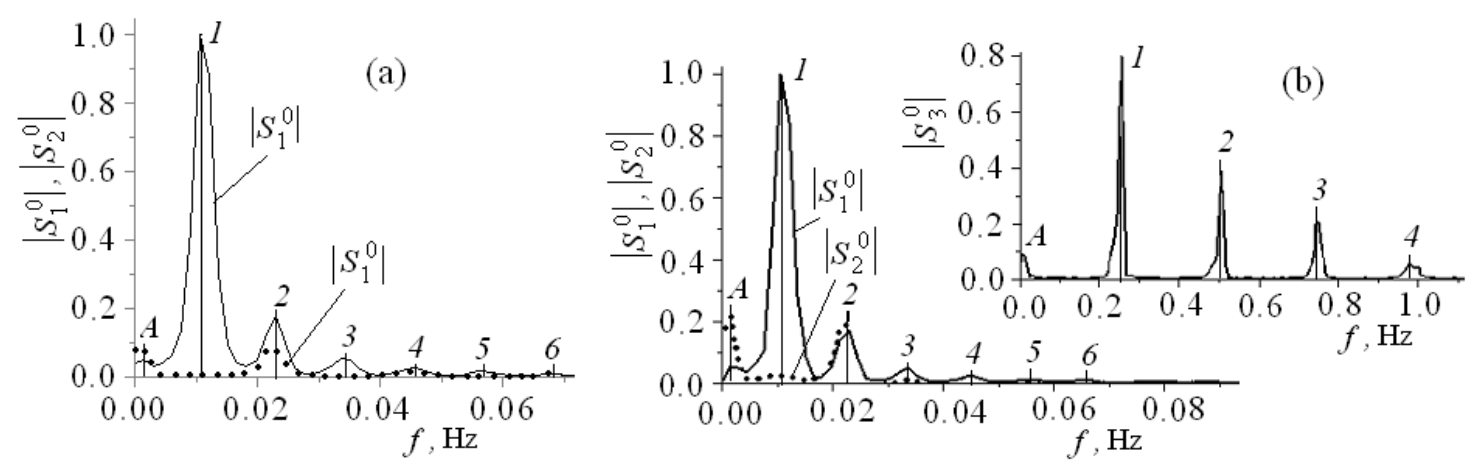

Figure 4. Modules of the spectra of the oscillations $\zeta_{i}$ shown in Figure 2

Notes: (a) Experiment 1: (b) Experiment 2.

The results of analysis of the correlation between the experimental frequencies $f_{j}^{e}$ and the theoretical frequencies $f_{p}=\omega_{p} / 2 \pi$ are presented in Figure 5. The following new symbols are used in Figure 5: $f_{p}^{i n}$ is the frequency of the theoretical internal seiche (Figure 5(a)) and $f_{p}^{\text {sur }}$ is the frequency of the theoretical surface seiche (Figure 5(b)). The condition $p=j$ is satisfied for the discrete points in Figure 5. Formula (8) gives almost the same results as formula (6) in the considered example. This is also true with respect to formulae (7) and (5).

If the correlation is ideal, the discrete points to be grouped around the coordinate angle bisector which is shown by the straight line in Figure 4. The data presented in Figure 4 show that the correlation is practically ideal at least up to $p=10$ when the strict formulae (3) and (4) are used. The approximate formulae (5) and (6) give acceptable results up to $p=5$ only. This is natural since they are based on the long wave approximation. Approximate formula (9) is acceptable in a wider range of the interfacial seiche natural frequencies.
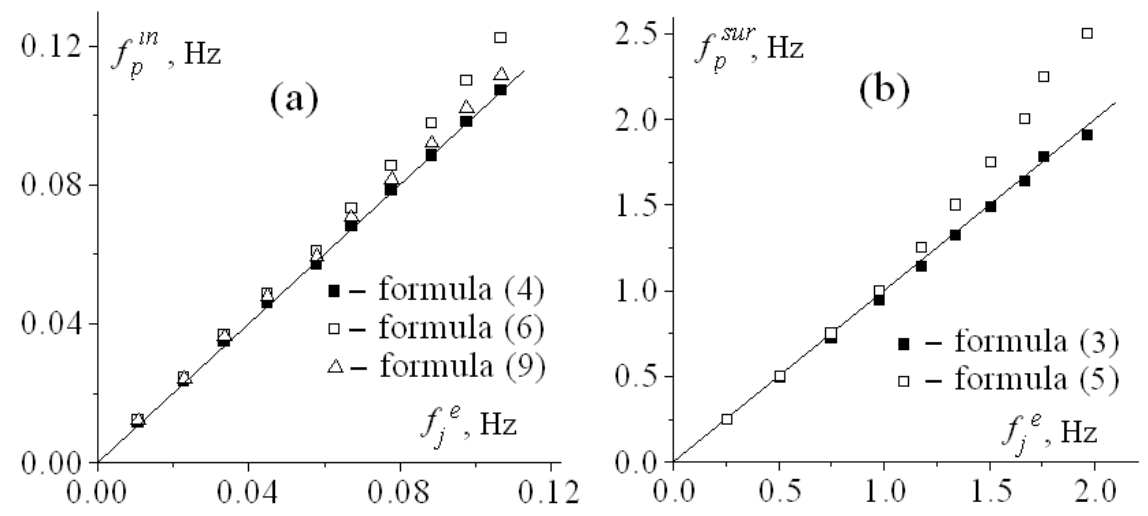

Figure 5. Correlation of the experimental (abscissa) and theoretical (ordinate) frequencies Notes: (a) Internal wave $\zeta_{1}$ in Experiment 1; (b) Surface wave $\zeta_{3}$ in Experiment 2. 


\section{Al Macrothink}

Aquatic Science and Technology

ISSN 2168-9148

2013, Vol. 1, No. 2

One result of the wave degradation analysis is presented in Figure 6. The interfacial oscillations $\zeta_{1}(t)$ near the basin right butt end in Example 1 was used in the analysis. The upper $Z_{u}(t)$ and lower $Z_{l}(t)$ envelopes and the oscillations $\zeta_{1}(t)$ are shown in Figure 6. The envelopes were described well enough by the following function:

$$
Z=E \exp \left(-t / t_{0}\right)
$$

where $E$ and $t_{0}$ are parameters of the $Z(t)$ function. They depend on the given parameters of the problem. The value $\alpha=1 / t_{0}$ is called the attenuation coefficient in the oscillation theory. In the example considered, $E=11.1 \mathrm{~mm}, t_{0}=118 \mathrm{~s}\left(\alpha=0.00847 \mathrm{~s}^{-1}\right)$ for the upper envelope and $E=-13.2 \mathrm{~mm}, t_{0}=130 \mathrm{~s}\left(\alpha=0.00769 \mathrm{~s}^{-1}\right)$ for the lower envelope.

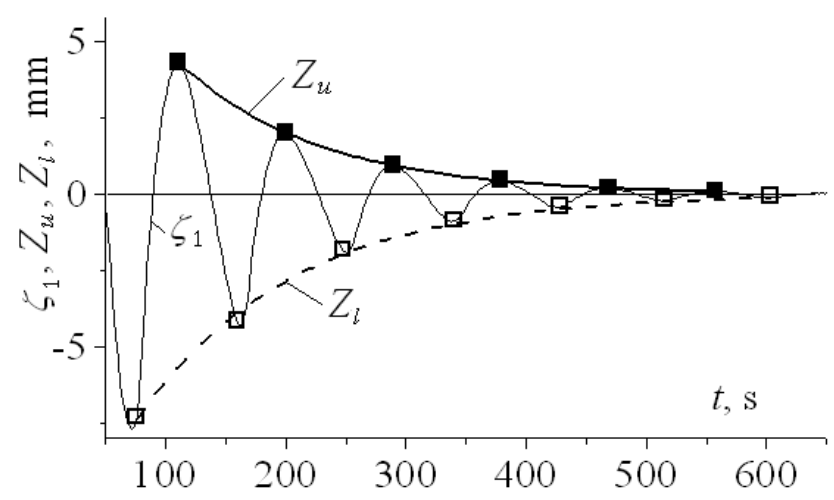

Figure 6. Envelopes of the internal wave $\zeta_{1}$ in Experiment 1

The surface seiches attenuated faster than the internal ones. For example, the parameters of the upper envelope of the oscillations $\zeta_{3}(t)$ in Example 2 (see Figure 2(b)) were the following: $E=13.9 \mathrm{~mm}, t_{0}=26 \mathrm{~s}$, i.e., the surface seiche attenuated approximately 4.5 times faster than the internal one.

\section{Discussion}

Natural water bodies have very complex geometry so any simplification of mathematical models of seiches is desirable. The long-wave approximation is used in all examples of natural seiche numerical computations (Dotsenko \& Miklashevskaya, 2010; Terletska et al., 2011). The results of the present study confirm the validity of this approximation. As the data given in Figure 4 show, this approximation is good for internal waves up to the frequency $\omega=2 \pi f \leq 0.3(\mathrm{rad} / \mathrm{s})$ at last. In the accordance with dispersion relation (1), the corresponding range of wave numbers is $k \leq 6.4\left(\mathrm{~m}^{-1}\right)$ and the corresponding range of wavelengths is $(\lambda=2 \pi / k) \geq 1(\mathrm{~m})$. The same value is also approximately true for the surface waves. Natural seiches are much longer than $1 \mathrm{~m}$.

The good coincidence of the experimental and strict theoretical frequencies in Figure 4 justifies the most significant simplification of the theory, namely: using the potential motion 
model. From this result, it follows that the fluid viscosity did not affect the length and frequency of the seiche even in the laboratory experiment. The theoretical and experimental study of the water viscosity influence on the dispersion relation for a two-layer fluid was performed in the work of Borodina et al. (1982). It has been shown that this influence is noticeably manifested only at the wavelengths less than $1.5 \mathrm{~cm}$.

\section{Conclusions}

The validity of the linear theory of potential motion at computations of the wavelengths and oscillation frequencies of the surface and internal seiches is confirmed by the example of a two-layer fluid. Relevancy of the long-wave approximation in the mentioned theory for natural seiches is also confirmed. Calculations of the seiche amplitudes, in particular, their attenuation, should be performed on the base of a mathematical model that takes into account specific disturbances and dissipation of energy.

\section{Acknowledgement}

This work was supported by Presidium of Russian Academy of Science (scientific program no. 2.13.3) and Presidium of Siberian Branch of this Academy (scientific program no. 132).

\section{References}

Boegman, L. (2004). The degeneration of internal waves in lakes with sloping topography. (Doctoral dissertation). The University of Western Australia, p. 146. Retrieved from http://www.civil.queensu.ca/Research/Hydrotechnical/Leon-Boegman/documents/boegmanp hd.pdf

Boegman, L., Ivey, G. N., \& Imberger, J. (2005). The energetics of large-scale internal wave degeneration in lakes. Journal of Fluid Mechanics, 531, 159-180. http://dx.doi.org/10.1017/S0022112005003915

Borodina, N. N., Bukreev, V. I., Gusev, A. V., \& Sturova, I. V. (1982). Viscosity attenuation of internal waves emerging in two-layer fluid at a movement of a cylinder and wing. Dinamika sploshnoy sredy (Dynamics of continuum), Issue 54. Institute of hydrodynamics: Novosibirsk, 49-60. (in Russian) http://dx.doi.org/10.1007/s11110-010-9075-1

Bukreev, V. I. (2011). Decay of an initial discontinuity of water depth in a finite-length channel. Experiment. Journal of Applied Mechanics and Technical Physics, 52(5), 689-697. http://dx.doi.org/10.1134/S0021894411050026

Dotsenko, S. F., \& Miklashevskaya, N. A. (2010). Baroclinic seiches in rotating basins of variable depth in the case of two-layer density stratification. Physical Oceanography, 20(3), 157-169.

Horn, D. A., Imberger, J., \& Ivey, G. N. (2001). The degeneration of large-scale interfacial gravity waves in lakes. J. Fluid Mech., 434, 181-207. http://dx.doi.org/10.1017/S0022112001003536

Kochin, N. E., Kibel’, I. A., \& Roze, N. V. (1963). Theoretical Hydromechanics (Chapter 1) 
Moscow: Fizmatgiz. (in Russian)

Ovsyannikov, L. V., Makarenko, N. I., Nalimov, V. I., et al. (1985). Nonlinear problems of the surface and internal wave theory (p. 318). Novosibirsk: Nauka, Siberian division. (in Russian)

Phillips, O. M. (1967). The dynamics of the upper ocean (p.270). Cambridge: Cambridge Univ. Press.

Rabinovich, A. B. (2009). Seiches and Harbor Oscillations. In Y.C. Kim (Ed.) Handbook of Coastal and Ocean Engineering (Chapter 9, pp. 194-236). Singapoure: World Scientific Publ.

Terletska, K. V., Maderich, V. S., \& Brovchenko, I. O. (2011.) Strongly nonlinear internal seiches in the elongated stratified lakes and phenomenon of "Loch Ness monster". Prikladna hidromehanika, 13(1), 51-55.

Thorpe, S. A. (1971). Experiments on the instability of stratified shear flows: miscible fluids. J. Fluid Mech., 46, 299-319. http://dx.doi.org/10.1017/S0022112071000557

\section{Symbols}

A Designation of one of maxima in Figure 3

$B$ Channel width (m)

$b_{p}$ Dimensionless parameter

$c_{p}$ Dimensionless parameter

E Parameter (mm)

$f$ Frequency $(\mathrm{Hz})$

$f_{p}$ Theoretical normal frequency $(\mathrm{Hz})$

$f_{p}^{\text {in }}$ Theoretical normal frequency of internal mode $(\mathrm{Hz})$

$f_{p}^{\text {sur }}$ Theoretical normal frequency of surface mode $(\mathrm{Hz})$

$f_{j}^{e}$ Frequency of experimental maximum $(\mathrm{Hz})$

$g$ Acceleration due to gravity $\left(\mathrm{m} / \mathrm{s}^{2}\right)$

$H$ Initial overall depth of fluid (m)

$h_{1}, h_{2}$ Initial depths of the upper and lower fluids respectively (m)

$i=1,2,3 \quad$ Index

$j=1,2,3, \ldots \quad$ Index 


\section{Macrothink}

$k$ Wave number $\left(2 \pi \cdot \mathrm{m}^{-1}\right)$

$k_{p}$ Normal wave number $\left(2 \pi \cdot \mathrm{m}^{-1}\right)$

$L \quad$ Basin length (m)

l Longitudinal coordinate of support (m)

$p=1,2,3, \ldots$ Index

$S_{i} \quad \operatorname{Spectrum}(\mathrm{mm} \cdot \mathrm{s})$

$\left|S_{1}^{0}\right|,\left|S_{2}^{0}\right|,\left|S_{3}^{0}\right| \quad$ Modules of individual dimensionless spectra

$t$ Time (s)

$t_{0} \quad$ Characteristic attenuation time (s)

$x \quad$ Longitudinal coordinate (m)

z Vertical coordinate (m)

$\alpha$ Attenuation coefficient $\left(\mathrm{s}^{-1}\right)$

$\beta$ Angle (degree)

$\delta \quad$ Characteristic thickness of intermediate layer (mm)

$\varepsilon \quad$ Dimensionless parameter

$\gamma$ Dimensionless parameter

$\lambda$ Wavelength (m)

$\rho_{1}, \quad \rho_{2}$ Densities $\left(\mathrm{kg} / \mathrm{m}^{3}\right)$

$\tau$ Characteristic time of rotation (s)

$\omega$ Circular frequency (rad/s)

$\omega_{p}$ Normal circular frequency (rad/s)

$\omega 1_{p}$ Normal circular frequency of surface mode (rad/s z)

$\omega 2_{p}$ Normal circular frequency of internal mode (rad/s)

$\zeta$ Vertical oscillation (mm)

$\zeta_{i}$ Vertical oscillations registered by wavegauges (mm)

$\zeta_{1}, \zeta_{2}, \zeta_{3}$ Individual oscillations (mm)

$\Theta_{1}, \Theta_{2}$ Dimensionless parameters

$Z$ Envelope (mm) 


\section{Macrothink}

$Z_{u}, Z_{l} \quad$ Upper and lower envelopes (mm)

\section{Copyright Disclaimer}

Copyright reserved by the author(s).

This article is an open-access article distributed under the terms and conditions of the Creative Commons Attribution license (http://creativecommons.org/licenses/by/3.0/). 